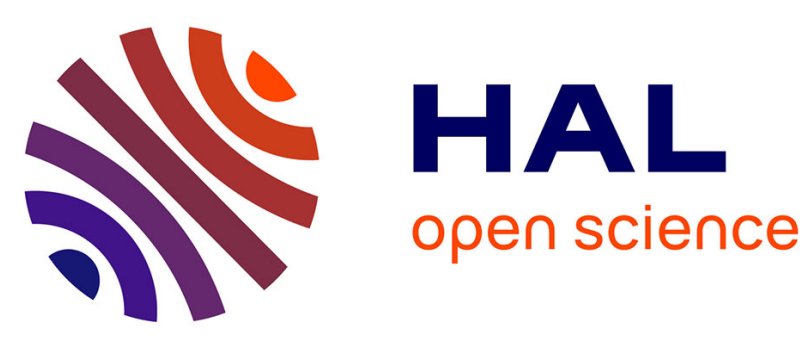

\title{
Effect of high-energy mechanical milling on the FSDP-related XRPD correlations in Se-rich glassy arsenic selenides
}

Y. Shpotyuk, Catherine Boussard-Plédel, B. Bureau, P. Demchenko, J. Szlęzak, J. Cebulski, Z. Bujňáková, P. Baláž, O. Shpotyuk

\section{To cite this version:}

Y. Shpotyuk, Catherine Boussard-Plédel, B. Bureau, P. Demchenko, J. Szlęzak, et al.. Effect of highenergy mechanical milling on the FSDP-related XRPD correlations in Se-rich glassy arsenic selenides. Journal of Physics and Chemistry of Solids, 2019, 124, pp.318-326. 10.1016/j.jpcs.2018.09.036 . hal01905111

HAL Id: hal-01905111

https://hal-univ-rennes1.archives-ouvertes.fr/hal-01905111

Submitted on 9 Nov 2018

HAL is a multi-disciplinary open access archive for the deposit and dissemination of scientific research documents, whether they are published or not. The documents may come from teaching and research institutions in France or abroad, or from public or private research centers.
L'archive ouverte pluridisciplinaire HAL, est destinée au dépôt et à la diffusion de documents scientifiques de niveau recherche, publiés ou non, émanant des établissements d'enseignement et de recherche français ou étrangers, des laboratoires publics ou privés. 


\title{
correlations in Se-rich glassy arsenic selenides
}

\author{
Ya. Shpotyuk ${ }^{1,2}$, C. Boussard-Pledel ${ }^{3}$, B. Bureau ${ }^{3}$, P. Demchenko ${ }^{2}$, J. Szlęzak ${ }^{1,3}$, J. Cebulski ${ }^{1}$, \\ Z. Bujňáková ${ }^{4}$ P. Baláž ${ }^{4}$ O. Shpotyuk ${ }^{5,6^{*}}$ \\ ${ }^{1}$ Centre for Innovation and Transfer of Natural Sciences and Engineering Knowledge, \\ University of Rzeszow, 1, Pigonia str., 35-959, Rzeszow, Poland \\ ${ }^{2}$ Ivan Franko National University of Lviv, 1, Universytetska str., 79000, Lviv, Ukraine \\ ${ }^{3}$ Equipe Verres et Céramiques, Université de Rennes 1, 35042 Rennes Cedex, France \\ ${ }^{4}$ Institute of Geotechnics of Slovak Academy of Sciences, \\ 45, Watsonova str., 04001, Košice, Slovakia \\ ${ }^{5}$ Jan Dlugosz University in Czestochowa, 13/15, al. Armii Krajowej, Czestochowa, 42200, Poland \\ ${ }^{6}$ O.G. Vlokh Institute of Physical Optics, 23, Dragomanov str., Lviv, 79005, Ukraine
}

* the corresponding author olehshpotyuk@ yahoo.com

\begin{abstract}
The X-ray powder diffraction (XRPD) patterns related to the first sharp diffraction peak (FSDP) are registered for Se-rich $\mathrm{As}_{\mathrm{x}} \mathrm{Se}_{100-\mathrm{x}}$ glasses $(5 \leq \mathrm{x} \leq 40)$, subjected to high-energy mechanical milling in a dry mode with $500 \mathrm{~min}^{-1}$ rotational speed. The results are treated in terms of structural model assuming broad diffraction halos in the XRPD patterns appeared due to remnants of inter-planar "quasi-crystalline" correlations, disturbed by overlapping with diffuse halos originated from strongest inter-atomic correlations, in part, the nearest-neighbor correlations between cation-like atoms. Milling does not influence the FSDP in a vicinity of glassy $\mathrm{Se}(\mathrm{x}<20)$, while causes increase in the FSDP width $\Delta Q$ (the reduced correlation length $L$ ) in the glasses with $20 \leq \mathrm{x}<40$, these changes being ascribed to disturbed inter-planar arrangement in covalent-bonded network due to structural defects generated under attrition ball milling. Assuming contribution of the Ehrenfest diffraction from coordination spheres in the detected XRPD patterns, the latter is ascribed to broadening in the distribution of characteristic FSDP-related inter-atomic distances, thus testifying in a favor of more defective structure of milled glass.
\end{abstract}

Key words: glass; arsenic selenide; mechanical milling; X-ray powder diffraction; first sharp diffraction peak. 
The high-energy mechanical milling (MM) is known to be an effective technological tool allowing substances preparation in a variety of their nanostructurized states [1-3]. Most commonly, this technology is applied to crystalline solids undergoing MM-driven nanonization, often accompanied by different phase transformations, including crystalline-to-amorphous transition (vitrification) due to large amount of mechanically-generated defects [3]. Nevertheless, the influence of MM on structurally disordered substances was relatively rare addressed.

Recently, numerous experiments probing vitrification dynamics in the nanostructurized glasses clearly demonstrates surface effects, revealed as negative deviations from bulk glass-transition temperature $T_{g}$ (that is the $T_{g}$ depression [4-7]) and accelerated physical ageing (see, e.g., ref. [8] and literature therein). This phenomenon was ascribed to liquid-like layer at the glass surface, which diverges as $T_{g}$ is approached from below [4,5], while more microscopic picture of responsible transformations remains rather unclear.

In this work, we report first experimental results concerning MM effect on the intermediaterange structure of Se-rich glassy $\mathrm{g}-\mathrm{As}_{\mathrm{x}} \mathrm{Se}_{100-\mathrm{x}}$, taken from domain of their pronounced network glass-forming ability [9-11], including boundary compositions of stoichiometric g- $\mathrm{As}_{2} \mathrm{Se}_{3}$ (i.e. g$\left.\mathrm{As}_{40} \mathrm{Se}_{60}\right)$ and $\mathrm{g}-\mathrm{As}_{5} \mathrm{Se}_{95}$.

\section{Experimental}

The studied alloys were prepared using coarse-grained pieces of bulk $\mathrm{g}-\mathrm{As}_{\mathrm{x}} \mathrm{Se}_{100-\mathrm{x}}(\mathrm{x}=5,10,15$, $17.5,20,22.5,25,29,33,35,37,40)$ preliminary synthesized from high-purity elemental precursors (As and Se of 5N purity acquired in Sigma Aldrich, USA) as described elsewhere [9-12]. The synthesized alloys were completely amorphous, as it follows from their X-ray powder diffraction (XRPD) patterns showing only broad diffuse halos proper to glassy substances, conchlike fracture of fresh-ingot cut sections and far IR transparency [9]. The plane-parallel plates (1.0$1.5 \mathrm{~mm}$ in a thickness) cut from thermally-annealed ingots (annealed at $T_{a}$ temperature of $15-20^{\circ} \mathrm{C}$ below glass transition point $T_{g}$ ) were used as reference samples for input characterization. The densities $\rho\left( \pm 0.005 \mathrm{~g} \cdot \mathrm{cm}^{-3}\right)$ measured at room temperature by the Archimedes displacement technique in ethanol using the Mettler Toledo analytical balances, and mid-onset glass transition temperatures $T_{g}\left( \pm 3{ }^{\circ} \mathrm{C}\right)$ determined from DSC scans performed under $10 \mathrm{~K} / \mathrm{min}$ heating rate using TA Instrument Q20 calorimeter (Table 1), testify in favor of full adequacy of synthesized glasses with known counterparts from As-Se system [9-11].

The MM was performed in a dry mode using Pulverissete 6 mill (operated at $500 \mathrm{~min}^{-1}$ rotational speed and protective argon atmosphere) for $20 \mathrm{~min}$ in $250 \mathrm{~mL}$ tungsten carbide chamber (loaded with 50 tungsten carbide balls each having $10 \mathrm{~mm}$ in a diameter), using $\sim 3 \mathrm{~g}$ of coarse-grained glass of each composition preliminary sieved under $200 \mu \mathrm{m}$. The high-energy MM in these conditions 
does not change principally the amorphous state of the milled substances, as it follows from diffuse halos observed in their XRPD profiles. The particle sizes in the powders were detected by photon cross-correlation spectroscopy with Nanophox particle size analyzer (Sympatec) [13,14]. For each MM-affected sample, the typical single modal distribution was detected with x50 close to $180 \mathrm{~nm}$ (meaning that $50 \%$ of particles were smaller than $180 \mathrm{~nm}$ ) and x99 close to $270 \mathrm{~nm}$ (meaning that $99 \%$ of particles were smaller than $270 \mathrm{~nm}$ ), as it exemplified in Fig. 1 for milled g-As ${ }_{40} \mathrm{Se}_{60}$.

The identity of the milled phase was clarified by the XRPD analysis applied to the first sharp diffraction peak (FSDP), which is believed to be a signature of specific structural entities in glassy substances forming a so-called intermediate-range ordering over scale of a few tens of $\AA[15,16]$. The XRPD data were collected in transmission mode on a STOE STADI P diffractometer [17] with linear position-sensitive detector $\left(\mathrm{CuK \alpha} \alpha_{1}\right.$-radiation, curved Ge (111) monochromator on primary

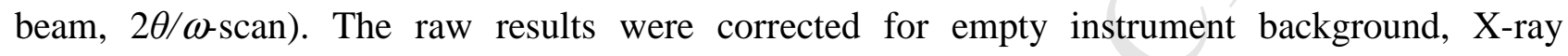
polarization, sample absorption, multiple and Compton scattering, Laue diffuse scattering and weighting correction, to obtain the total scattering structure function $S(Q)$, and its expression as the reduced scattering structure function, $F(Q)=Q[S(Q)-1]$, where $Q$ is the magnitude of the scattering vector [18]. The X-ray structure factors $F(Q)$ were obtained using the PDFgetX2 software package [19], the accuracy of $S(Q)$ calculation according to standard deviations in output-files being $\pm 0.5 \%$.

Preliminary data processing was performed with STOE WinXPOW [17] and PowderCell [20] program packages, using information on crystal structures of arsenic selenides taken from known databases [21,22], in part, the JCPDS card No. 65-2365 for monoclinic $\mathrm{As}_{2} \mathrm{Se}_{3}$ and card No. 730465 for trigonal Se. The processing of the experimental XRPD profiles was performed using these programs, following normalization procedure taken in respect to the intensity of maximum peak. Then, the obtained data were used for next profile fitting exploring the WinPLOTR program [23]. Under used fitting protocol, we can compare correctly effects of MM on the halos-structured XRPD patterns of compositionally different g-As-Se samples, parameterized in peak angular position $2 \theta$ and full width at half maximum (FWHM) defined within $\pm 0.05^{\circ} 2 \theta$ accuracy. The corresponding values of scattering vector $Q$ and width $\Delta Q$ in a reciprocal space were calculated as:

$$
\begin{aligned}
& Q=(4 \pi / \lambda) \cdot \sin \theta, \\
& \Delta Q=(4 \pi / \lambda) \cdot \sin (\mathrm{FWHM} / 2) .
\end{aligned}
$$

The characteristic distance $R$ (viz. the spacing of "quasi-periodicity" responsible for the FSDP, accepted this peak as a signature of remnants of crystallographic inter-planar periodicity) and correlation length $L$ over which this "quasi-periodicity" is maintained were calculated as [24-33]:

$$
\begin{aligned}
& R=2 \pi / Q, \\
& L=2 \pi / \Delta Q .
\end{aligned}
$$



arising from coordination spheres, i.e. groups of closest inter-atomic distances like in randomlypacked multiparticle systems [34-36], when scattering is governed by the Ehrenfest equation [37]:

$$
2 d_{s} \cdot \sin \theta=1.23 \cdot \lambda,
$$

where $d_{s}$ is an average inter-atomic distance in a structure (i.e. distance between scattering centers defined as radius of corresponding coordination sphere).

\section{Results and discussion}

For a long time during last decades, the compositional variations in the FSDP-related XRPD patterns in binary g-As-Se have been in a sphere of tight interests, since these disordered substances are archetypal representatives for whole class of covalent-network glass formers [9-11]. Deviations in the FSDP position, intensity and width were subjected to scrupulous and systematic research in [31-33], showing perfect correlation with changing in g-As-Se composition.

The experimental XRPD patterns for both unmilled and milled $\mathrm{g}-\mathrm{As}_{\mathrm{x}} \mathrm{Se}_{100-\mathrm{x}}$ demonstrate broad diffuse peaks ("amorphous" halos) proper to g-As-Se/S [25-28]) revealed in a vicinity of $\sim 15-22$, $\sim 28-32$ and $\sim 50-60^{\circ} 2 \theta$. In a reciprocal space, these halos can be scaled in respect to $Q \cdot d_{s}$ parameter (where $d_{s}$ denotes an averaged inter-atomic distance in glass network) positioned at $\sim 2-3, \sim 4.6-4.9$, 7.7-8.9 [26-29]. According to the Fourier-transform analysis [30], in a case of network-forming glassy materials, each of these features corresponds to a real-space ordering with characteristic distance $R$ ("quasi-periodicity") and correlation length $L$ defined respectively by eqs. (1) and (2). For nearest inter-atomic distances of $d_{s} \cong 2.2-2.6 \AA$ typical for glassy arsenic chalcogenides (e.g., $d_{s}$ $\cong 2.43 \AA$ for $\mathrm{g}-\mathrm{As}_{2} \mathrm{Se}_{3}[9-11]$ ), the first of these halos, associated with length scales commensurable with intermediate-range ordering of glass-forming structural motifs, is reproduced in the structure factor as the FSDP positioned at $Q \cong \sim 1-1.5 \AA^{-1}$. Under the same inter-atomic distances $d_{s}$, the halo at $\sim 28-32{ }^{\circ} 2 \theta$ (near $Q \sim 1.8-2.2 \AA^{-1}$ ) referred to as principal diffraction peak (PDP) [29] is ascribed to size of local network-forming motifs, which are deterministic for so-called extended-range ordering in a glass [15]. Correspondingly, the third peak at $\sim 50-60{ }^{\circ} 2 \theta$ associated with nearest-neighbor separations in a glass [29] corresponds to scattering vectors $Q \sim 3.3-4.0 \AA^{-1}$.

The XRPD profiles collected for some unmilled and milled $\mathrm{g}-\mathrm{As}_{\mathrm{x}} \mathrm{Se}_{100-\mathrm{x}}$ specimens as compared with calculated ones are shown in Fig. 2 and 3, respectively. The same features are also revealed in low- $Q$ part of the reduced X-ray structure factor $F(Q)=Q(S(Q)-1)$ depicted in Fig. 4. The FSDP parameters calculated for all these samples are gathered in Table 2.

It is seen that with increase in Se content (decrease in $x$ parameter), the FSDP position in $\mathrm{g}$ $\mathrm{As}_{\mathrm{X}} \mathrm{Se}_{100-\mathrm{x}}$ shifts to higher scattering vectors $Q$, these values for unmilled samples being in an excellent agreement with data of other authors [31-33]. Simultaneously, under decreased $x$ parameter, the FSDP is affected to substantial broadening in the width $\Delta Q$. In the structure factor 
$F(Q)=Q(S(Q)-1)$ determination, the FSDP parameters of unmilled $\mathrm{g}-\mathrm{As}_{2} \mathrm{Se}_{3}$ reach $Q=1.28 \AA^{-1}$ and $\Delta Q=0.43 \AA^{-1}$ (Fig. 4), while the PDP observed at $2.23 \AA^{-1}$ is wider in a width $\left(0.54 \AA^{-1}\right)$. With increase in Se content beyond $\mathrm{As}_{2} \mathrm{Se}_{3}$ composition ( $\left.x=40\right)$, the FSDP becomes merely reduced in its magnitude and broaden in a width, being shifted towards higher scattering vectors $Q$ (so demonstrating high- $Q$ shift seen from Fig. 4). Under this trend, the PDP remotes in an opposite direction demonstrating very strong low- $Q$ shift. Thus, approaching g-Se in g-As-Se, the intermediate-range ordering determined by the FSDP is depressed being covered by extended-range ordering due to low- $Q$ shifted PDP at $\sim 26-28{ }^{\circ} 2 \theta$ (corresponding to $Q \sim 1.6-1.7 \AA^{-1}$ ).

The high-energy MM does not change principally an overall character of these XRPD patterns. Thus, in the milled $\mathrm{g}-\mathrm{As}_{\mathrm{x}} \mathrm{Se}_{100-\mathrm{x}}(5 \leq \mathrm{x} \leq 40)$, the FSDP position $Q$ and, respectively, the spacing of the FSDP-responsible "quasi-periodicity" $R$ remains nearly the same as in unmilled samples (Table 2). In contrast, the FSDP width $\Delta Q$ is slightly enhanced under MM and, respectively, the correlation length $L$ is more reduced, this effect being observed for milled g-As $S_{x} \mathrm{Se}_{100-\mathrm{x}}$ samples with $20 \leq \mathrm{x}<40$. The relative changes in the correlation length $\Delta L / L_{0}$ calculated in respect to unmilled samples reach 5-6\%, while changes in the periodicity $\Delta R / R_{0}$ remains within measuring error-bar (no more $0.8 \%$ ), except close to stoichiometric glass compositions with $40 \leq x<35$ (see Table 2). These effects are not accompanied by changes in the PDP (neither in peak position $Q$ nor in width $\Delta Q$ ), speaking in a favor of MM-driven modification of intermediate-range ordering alone.

Despite controversies concerning the FSDP origin in covalent-bonded glass formers, this peak is generally accepted as a manifestation of intermediate-range order in a glass structure $[15,16]$. Recently the Gaskel's concept on crystalline-like remnants in melt-quenched (MQ) glasses [38] modified with Wright's justification for the FSDP as arising from a periodicity in the distribution of some "pseudo-planes" for the Bragg diffraction separating succession of randomly-packed network cages [39], was applied to interpret crystalline-to-amorphous transition in arsenic chalcogenides affected to high-energy MM [40,41]. In respect to this concept, the flattened cage structure of covalent-bonded network glasses produces inner equivalent planes at opposite sides of these cages forming a necessary contribution to the reciprocal space Fourier component generating the FSDP [39]. Therefore, the FSDP is ascribed to variations in the underlying cage structure of glassy network defined by interlinked polyhedrons (glass-forming building blocks) producing multiple cycle-type entities, which keep some elements of inter-planar "ordering" proper to crystalline counterparts of these glasses of close chemical compositions.

Within this concept $[38,39]$ applied to $g-\mathrm{As}_{\mathrm{x}} \mathrm{Se}_{100-\mathrm{x}}(5 \leq \mathrm{x} \leq 40)$, the FSDP can be attributed to covalent-bonded network structure built of trigonal $\mathrm{AsSe}_{3 / 2}$ pyramids interlinked by Se-chains, i.e. inter-planar "ordering" character for crystalline $\mathrm{As}_{2} \mathrm{Se}_{3}$ (with specific 12-membered corrugated rings within $a c$ plane $[9,42-44])$, disturbed by incorporated (-Se-Se-) $)_{n}$ chains of different length $n$ (dependently on a glass composition) [45-54]. With Se additions to stoichiometric g- $\mathrm{As}_{2} \mathrm{Se}_{3}$, the 
FSDP-responsible inter-planar ordering does not change essentially. The higher content of homonuclear Se-Se bonds in a glassy network, which are shorter than other covalent chemical bonds in g-As-Se $\left(l_{S e-S e} \cong 2.32 \AA, l_{A s-S e} \cong 2.43 \AA\right.$, $l_{A s-A s} \cong 2.53 \AA$ [9-11]), the shorter average inter-atomic distance $d_{s}$, and, respectively, the smaller "quasi-periodicity" $R$, while the correlation length $L$ decreases only slightly (see Table 2). These changes are accompanied by dropping in the FSDP intensity, this effect being accompanied by evident increase in the $L$ with Se addition when going over a barrier of $\sim 80$ at. $\%$ Se. The boundary composition (i.e. g- $\mathrm{As}_{20} \mathrm{Se}_{80}$ ) corresponding to the eutectic in the phase diagram of As-Se system [9,11,52,53] can be specified as a threshold between network of $\mathrm{AsSe}_{3 / 2}$ pyramids linked by (-Se-Se-) $)_{n}$ chains with an essential role of cation-cation correlations, and network of Se-rich entities in preferential trans- (Se-chains) and/or cis-atomic configurations (Se-rings) $[39,53,54]$. In the latter, the cation-cation correlations responsible for the FSDP [31] became inessential diminishing this peak with further Se content.

Under high-energy MM, the existing thermodynamically stabilized balance in network-forming entities is essentially disturbed, providing an evidence on their defective states with unfavorable potential energies. From inspection of Table 2, it seems that MM does not influence the FSDPresponsible correlations in $\mathrm{g}-\mathrm{As}_{\mathrm{x}} \mathrm{Se}_{100-\mathrm{x}}$ close to $\mathrm{g}$-Se $(5<\mathrm{x}<20)$, where Se-rich entities are obviously dominated in the structural network. At the same time, slight increase in the FSDP width $\Delta Q$ for milled $g-\mathrm{As}_{\mathrm{x}} \mathrm{Se}_{100-\mathrm{x}}$ with $20 \leq \mathrm{x} \leq 40$ can be ascribed to reduction in the correlation length $L$ due to different structural defects generated under MM, which disturb the FSDP-responsible inter-planar "ordering" due to built-in attrition stress. This compositional domain in binary g-As-Se is known to be full of different structural-network instabilities, such as local decomposition on As- and Se-rich fragments or global separation on $\mathrm{As}_{2} \mathrm{Se}_{3}$-rich and Se-rich phases [54]. Such anomalies activated by ball MM occur fragmentation effect on the correlation length $L$ of the FSDP-responsible "quasiperiodicity".

In general, from the point of thermodynamic enthalpic diagram for glass-forming liquids [8,9], the MQ glassy substance is closer to the equilibrium state than that subjected to high-energy MM. This conclusion essentially depends on the cooling rate of melt liquid (at slow cooling rate, the near-equilibrium glasses dominate, while far-from-equilibrium glasses are preferred at rapid cooling) [9]. In such consideration, the milled substance is undoubtedly closer to out-of-equilibrium state of fine-grained powder. It should be expected that under increased energy transferred to solid system under high-energy MM (e.g., due to increased rotational speed or more prolonged milling), the most out-of-equilibrium state will be achieved because of large number of structural defects appeared at such conditions. Thermodynamic characteristics of amorphous solids derived from different states (melt, super-cooled liquid, or crystal) due to applied amorphization technique is expected to deviate as for extra-rapidly MQ glass affected to prolonged physical ageing, this effect being essentially dependent on glass composition $[8,9]$. 
patterns of g-As-Se can be presented accepting their diffuse halos as additionally disturbed by pair inter-atomic correlations, i.e. superimposed the Ehrenfest diffraction halos originated from coordination spheres (see, e.g., ref. [35] and cited literature therein). In a strong meaning, such approach alone is confirmed only for randomly-packed multiparticulate substances [34-36], which are classified in this view as ideal amorphous solids obeying geometrical structural model composed of contacting hard spheres in a space positioned in a random way [36]. In application to covalent-bonded substances (like chalcogenide glasses) possessing atomic-network structure comprising short- and intermediate-range ordering, this approach is not strictly applicable [24,36]. The governing role of the XRPD signatures attributed to "quasi-crystalline" inter-planar correlations in such a case can be credibly documented by close similarity between diffuse halos and strongest reflexes of crystalline homologs of these glasses of close compositions [55,56]. Thus, Fig. 5 demonstrates the XRPD profiles for unmilled and milled g-As ${ }_{40} \mathrm{Se}_{60}$ in comparison with theoretical Bragg diffraction reflexes for monoclinic $\mathrm{As}_{2} \mathrm{Se}_{3}$ and trigonal Se [21,22]. As stated above, the XRPD patterns of such substances contain a few spatially-separated diffuse halos at $\sim 50$ 60, $\sim 28-32$ and $\sim 15-22^{\circ} 2 \theta$, where third one is referred to as the FSDP [25-33]. With the Ehrenfest eq. (5), this peak-halo is ascribed to the X-ray diffraction from averaged inter-atomic distances $d_{s}$, changing smoothly from $6.05 \AA$ in g-As $_{40} \mathrm{Se}_{60}$ to 5.42 in $\mathrm{g}-\mathrm{As}_{5} \mathrm{Se}_{95}$ (Table 2). In g-As-Se, these $d_{s}$ correspond to character distances between cation-like atoms of some fragments belonging to different more or less separated atomic clusters (quasi-layers), forming third-order nearestneighbor (As)---(As) pairs correlations [15,16,31-33]. At least two of these inter-atomic distances prevail over others in glassy chalcogenides [9-11,57,58]. Thus, in the atomic arrangement of both glassy and crystalline $\mathrm{As}_{2} \mathrm{Se}_{3}$, the dominated $d_{s}$ approaching $\sim 5.8$ and $\sim 6.9 \AA$ terminated by deep depopulation regions can be well detected (see Fig. 3 in ref. [57]). In this glass, the lower $d_{s}$ is expected for right-sided wing of the FSDP (observed at higher diffraction angles $2 \theta$ ), while the longer distances are proper to left-sided part of this peak (observed at lower diffraction angles $2 \theta$ ).

Therefore, we believe the FSDP in $\mathrm{g}-\mathrm{As}_{\mathrm{x}} \mathrm{Se}_{100-\mathrm{x}}(5 \leq \mathrm{x} \leq 40)$ monitored at scattering vectors $Q \sim 1.28-1.42 \AA^{-1}$ can be attributed to commensurable contributions from (i) inter-planar correlations due to "quasi-crystalline" remnants of close compositions (monoclinic $\mathrm{As}_{2} \mathrm{Se}_{3}$ and trigonal $\mathrm{Se}$ ) with character Bragg diffraction lengths $R=4.92-4.43 \AA$, and (ii) inter-atomic correlations due to thirdorder nearest-neighbor (As)---(As) pairs with character $d_{s}=6.05-5.45 \AA$ (the digits are given in a sequence of increasing Se content, Table 2).

This multi-level structure-forming specificity is assumed to be a reason for anomalous behavior of the FSDP under compositional variations, applied pressure, temperature or other influences. Thus, e.g., if the inter-planar distance $R$ is decreased under some factors, such as applied pressure, thus resulting in high- $Q$ shift in the FSDP position, the accompanied changes in (As)---(As) inter- 
atomic distances $d_{s}$ are inadequate because of orientation displacements of neighboring structural fragments (molecular clusters or quasi-layers). Some inter-atomic distances $d_{s}$ occur to be increased at a cost of decreased others, both contributing to the same coordination sphere producing the XRPD halo near $Q \sim 1 \AA^{-1}$ (the FSDP). Under acentric displacements of constituting quasi-layers or molecules, this densification trend may result in somewhat decreased shorter distances $d_{s}$, but increased longer ones. Correspondingly, the increased inter-atomic distances remote from the FSDP-related contributions, depressing the FSDP intensity and shifting it towards shorter $d_{s}$, these changes being in excellent agreement with known experimental observations showing FSDP shifting to higher $Q$ accompanied by broadening and decaying under densification [16,59,60].

So we can speculate that under MM some inter-atomic distances $d_{s}$ contributing to the FSDP are destroyed owing to created defects, giving rise to others. Essential reduction of such inter-atomic (As)---(As) correlations in initial (unmilled) state as resulted from atomic pairs distributions derived by Renninger and Averbach [57], make $\mathrm{g}-\mathrm{As}_{\mathrm{x}} \mathrm{Se}_{100-\mathrm{x}}$ with $\mathrm{x}<20$ stable against MM (no detectable changes in the FSDP position $Q$ and width $\Delta Q$, Table 2). Presumably, main compositional trend in the FSDP position showing strong tendency to higher $2 \theta$ with Se content is governed by broadened inter-planar Bragg reflexes from crystalline phases, these being monoclinic $\mathrm{As}_{2} \mathrm{Se}_{3}[43,44]$ with

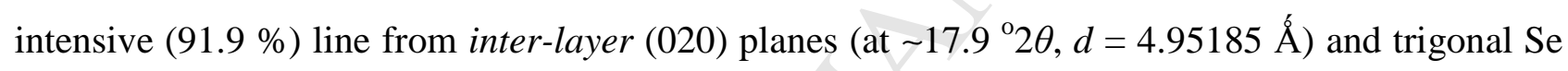
[61] with more than twice weaker (50\% in intensity) line from (100) planes at $\sim 23.5{ }^{\circ} 2 \theta$ corresponding to 3.78124 Á distance (Fig. 5).

As to the PDP positioned in $\mathrm{g}-\mathrm{As}_{2} \mathrm{Se}_{3}$ at $Q \sim 2.23 \AA^{-1}$ (which is equivalent to $\sim 31.7^{\circ} 2 \theta$ ) $[29,36]$ ), the responsible inter-atomic correlations $d_{s}$ for this peak calculated from the Ehrenfest eq. (5) reach for g-As-Se 3.6-4.0 Á, the values which can be ascribed to nearest-neighbor distances related to second-order (As)---(Se) and (Se)---(Se) atomic pairs. Such attributes can be found, in part, in the crystallographic projection of crystalline $\mathrm{As}_{2} \mathrm{Se}_{3}$ within centrosymmetrically equivalent composite polyhedrons around two As sites as defined by Stergiou and Rentzeperis [43]. That is why angular positions of both inter-planar "quasi-crystalline" $R$ and inter-atomic $d_{s}$ correlations contributing to this peak coincide merely. As to compositional trends followed from atomic radial distributions derived by Renninger and Averbach [56], the inter-atomic correlations at $d_{s} \sim 3.7 \AA$ are full invariant with Se content in g-As-Se. Therefore, the compositional changes in this region are governed by Bragg diffraction of "quasi-crystalline" inter-planar correlations, showing strong low- $Q$ shift with Se content due to preference of broad crystallographic reflexes of trigonal Se (Fig. 5), that is $100 \%$ in intensity (101) planes $\left(\sim 30.0{ }^{\circ} 2 \theta, d=3.00565 \AA\right)$ and $50 \%$ in intensity (100) planes $\left(\sim 23.5^{\circ} 2 \theta\right.$, $d=3.78124$ Á) [61]. Noteworthy, the PDP-related inter-atomic distances $d_{s}$ correlate well with mean inter-atomic spacing $l_{s}=\left(6 \cdot \mu / \pi \cdot \rho \cdot N_{A}\right)^{1 / 3}$ in Table $1\left(\mu-\right.$ molecular mass of glass, $N_{A}-$ the Avogadro's number) derived from macroscopic densities $\rho$ of g-As-Se. 

reproducible via the Ehrenfest eq. (5) as proper to mean inter-atomic distances of $d_{s}=2.0-2.1 \AA$ (which is less than character first-order nearest-neighbor bond lengths in binary g-As-Se), is most controversial in this interpretation. It should be emphasized that strict one-to-one correspondence within the Ehrenfest eq. (5) is appropriate only for amorphous substances obeying structural models built of hard contacting spheres like those proper to metallic glasses [24,36]. In semiconductor network covalent-bonded glasses, where these peaks originates from competition between rapidlydecreasing form-factor for separate structural units (building blocks like trigonal $\mathrm{AsSe}_{3 / 2}$ pyramids, $\mathrm{GeSe}_{4 / 2}$ tetrahedrons, etc.) and raising structure factor associated with their spatial packaging [62], this correspondence is not strictly satisfied due to directionality of covalent bonds preventing glass structure from close nearest-neighbor packing in the shortest coordination sphere. Respectively, the Ehrenfest relation (5) in its original form cannot be further applied as the Master equation covering realistic shortest inter-atomic distances $d_{s}$ in covalent-bonded systems without additional correction. At the basis of data for $\mathrm{g}-\mathrm{As}_{2} \mathrm{Se}_{3}$ gathered in Table 2, this correction can be simply enter with multiplying coefficient of $\sim 1.14$, thus giving remarkable correspondence with peak position $d_{s}$ attributed to As-Se bond length ( 2.43 $\AA$ ). The governing compositional trend in this peak-halo in Se-rich g-As-Se also corresponds to changes in mean inter-atomic spacing $l_{s}$ showing smooth increase towards Se-rich compositions (see Table 1).

It worth mentioning interpretation of the FSDP-related dependences in $\mathrm{g}$-As-Se in terms of XRPD patterns originated from remnants of "quasi-crystalline" inter-planar correlations supplemented by diffuse halos originated from strongest inter-atomic correlations in a glass network, has an obvious advantage over models treated these patterns as arising from "quasiperiodicity" of crystallographic planes only. Indeed, within this approach, we explain appearance of additional diffuse halos at smaller diffraction angles of $\sim 5-7^{\circ} 2 \theta$ (Fig. 2, 3 and 5) in the region without any close "quasi-crystalline" inter-planar reflexes (so-called pre-FSDP [63-67]) as corresponding to extended super-structural units, which reveal more prolonged inter-atomic correlations reaching $d_{s} \sim 15-20 \AA$. An original interpretation can be also provided for high-angular shoulder in the right-sided FSDP wing or so-called post-FSDP [63] observed at $\sim 20-22{ }^{\circ} 2 \theta$ (see Fig. 2, 3 and 5) or $Q \sim 1.5 \AA^{-1}$ (see Fig. 4). Such feature causing an essential asymmetry in the FSDP shape (as was pointed out by Ke. Tanaka for photo-expanded chalcogenide films [68]), was observed often in the XRPD patterns of many bulk glassy alloys (especially those doped with metals), such as $\mathrm{g}-\mathrm{As}_{2} \mathrm{~S}_{3}[28,69]$ and near-stoichiometric g- $\mathrm{As}_{38} \mathrm{~S}_{62}$ and $\mathrm{g}-\mathrm{As}_{42} \mathrm{~S}_{58}$ [69], $\mathrm{As}_{2} \mathrm{~S}_{3^{-}}$ In/I/GaSb [64,66], Ge-Sb-S [65], Ge-As-Se [67], $\mathrm{GeSe}_{2}-\mathrm{Ga}_{2} \mathrm{Se}_{3}$ [70,71], $\mathrm{As}_{2} \mathrm{Se}_{3}-\mathrm{Ga}$ [72], and even $\mathrm{As}_{45} \mathrm{~S}_{55}$ amorphized by high-energy MM [73]. To the best of our knowledge, this specificity has been remained without explanation, giving a ground for supposition on possible doublet-type constitution of the FSDP in chalcogenide glasses. Indeed, this shoulder corresponding to inter- 
atomic distances approaching $d_{s}=5 \cdot 1-5.3 \AA$ (irrespectively to glass composition), can be ascribed to the Ehrenfest diffraction from some remnants of "quasi-crystalline" correlations, which also contribute to the FSDP through more intensive Bragg reflection.

From a standpoint of the XRPD in chalcogenide glasses originated from coordination spheres, the MM-induced invariance in the FSDP positions $Q$ for $\mathrm{g}-\mathrm{As}_{\mathrm{x}} \mathrm{Se}_{100-\mathrm{x}}(5 \leq \mathrm{x} \leq 40)$ means unchangeable behavior of inter-atomic distances $d_{s}$ contributing to the FSDP-responsible coordination sphere (the maximal changes $\Delta d_{s} / d_{s 0}$ after milling do not exceed the reliable error-bar of $\pm 1.0 \%$, see Table 2). In more Se-rich glasses with $5 \leq x \leq 20$, this effect is concomitant with nearly constant FSDP width $\Delta Q$ (or peak FWHM) for unmilled and milled samples, this effect being ascribed to unchanged distribution of these inter-atomic distances $d_{s}$. In contrast, the glasses with $20 \leq \mathrm{x} \leq 40$ demonstrate slight increase in the FSDP width $\Delta Q$ after high-energy MM owing to broadening in the distribution of characteristic inter-atomic distances $d_{s}$ contributing to the FSDP, thus testifying in a favor of more destructed glass structure in the milled state.

\section{Conclusions}

The FSDP-related correlations are studied in the XRPD patterns of Se-rich $\mathrm{As}_{\mathrm{x}} \mathrm{Se}_{100-\mathrm{x}}$ glasses $(5 \leq x \leq 40)$, modified by high-energy mechanical ball milling in a dry mode with $500 \mathrm{~min}^{-1}$ rotational speed. The results are treated in terms of structural model assuming appearance of broad diffuse halos due to remnants of inter-planar "quasi-crystalline" correlations (in respect to the GaskelWright's concept on microcrystalline ordering in network glass-formers [38,39]), supplemented by halos originated from strongest inter-atomic correlations, in part, the nearest-neighbor correlations between cation-like atoms. It is shown that high-energy mechanical milling does not influence the FSDP position $Q$ in a vicinity of glassy $\operatorname{Se}(5<x<20)$, but increase the FSDP width $\Delta Q$ (reduction in the correlation length $L$ ) for $\mathrm{g}-\mathrm{As}_{\mathrm{x}} \mathrm{Se}_{100-\mathrm{x}}$ with $20 \leq x<40$, the latter being caused by disturbed interplanar arrangement due to generated structural defects. Alternatively, this effect is ascribed to broadening in the distribution of characteristic inter-atomic distances $d_{s}$ contributing to the FSDP, thus testifying in a favor of more destructed glass structure in the milled state.

\section{Acknowledgements}

This work was supported by the Slovak Research and Development Agency under the contract no. APVV-14-0103 and Slovak Grant Agency VEGA (project 2/0027/14). The paper contains results of studies conducted by the President's of Ukraine grant (F70/134-2017) of the State Fund for Fundamental Research.

\section{References}


Heidelberg, 2008.

2. Baláž P., Baláž M., Achimovicova M., Bujňáková Z., Dutkova E. Chalcogenide mechanochemistry in materials science: insight into synthesis and applications (a review). - J. Mater. Sci. 52 (2017) 11851-11890.

3. Baláž P., Achimovicova M., Baláž M., Billik P., Cherkezova-Zheleva Z., Manuel Criado J., Delogu F., Dutkova E., Gaffet E., Gotor F.J., Kumar R., Mitov I., Rojac T., Senna M., Streletskii A., Wieczorek-Ciurowa K. Hallmarks of mechanochemistry: from nanoparticles to technology. Chem. Soc. Rev. 42 (2013) 7571-7637.

4. Keddie J.L., Jones R.A., Cory R.A. Size-dependent depression of the glass transition temperature in polymer films. - Europhys. Lett. 27 (1994) 59-64.

5. Keddie J.L., Jones R.A., Cory R.A. Interface and surface effects on the glass-transition temperature in thin polymer films. - Faraday Disscuss. 98 (1994) 219-230.

6. Ellison C.J., Torkelson J.M. The distribution of glass-transition temperatures in nanoscopically confined glass formers. - Nature Mater. 21 (2003) 695-700.

7. Salez T., McGraw J.D., Dalnoki-Veress K., Raphael E., Forrest J.A. Glass transition at interfaces. - Europhys. News 48 (2017) 24-28.

8. Cangialosi D., Alegria A., Colmenero J. Effect of nanostructure on the thermal glass transition and physical ageing in polymer materials. - Progr. Polym. Sci. 54-55 (2016) 128-147.

9. Feltz A. Amorphous Inorganic Materials and Glasses. - VCH Publ., Inc. Weinheim-New YorkBasel-Cambridge-Tokyo, 1993.

10. Feltz A., Aust H., Blayer A. Glass formation and properties of chalcogenide systems XXVI: Permittivity and the structure of glasses $\mathrm{As}_{\mathrm{x}} \mathrm{Se}_{1-\mathrm{x}}$ and $\mathrm{Ge}_{\mathrm{x}} \mathrm{Se}_{1-\mathrm{x}} .-\mathrm{J}$. Non-Cryst. Sol. 55 (1983) 179190.

11. Borisova Z.U. Glassy Semiconductors. - Plenum Press, New York-London, 1981.

12. Adam J.-L., Zhang X. Chalcogenide Glasses: Preparation, Properties and Application. Woodhead Publ. Ser. in Electronic and Opt. Mater., Philadelphia-New Delhi, 2013.

13. Lämmle W. Nanometre particle sizing and stability measurement using a table-top PCCS system. - LabPlus Intern 19 (2005) 19-24.

14. Lämmle W., Wolfgang J., Kesten U. Nanometre particle sizing and stability measurement with table top PCCS. - NSTI-Nanotech 20074 (2007) 124-127.

15. Elliott S.R. Extended-range order, interstitial voids and the first sharp diffraction peak of network glasses. - J. Non-Cryst. Sol. 182 (1995) 40-48.

16. Tanaka K. Chalcogenide Glasses. - Reference Module in Materials Science and Materials Eng., 2016. Encyclopedia of Materials: Science and Technology (Second Ed.), 2001, 1123-1131.

17. Stoe WinXPOW (version 3.03). - Stoe \& Cie GmbH, Darmstadt, Germany, 2010. 
18. Egami T., Billinge S.J.L.) Underneath the Bragg Peaks, Volume 16, Second Ed.: Structural Analysis of Complex Mater. - Pergamon Materials Ser., 2013.

19. Qiu X., Thompson J.W., Billinge S.J.L. PDFgetX2: a GUI driven program to obtain the pair distribution function from X-ray powder diffraction data. - J. Appl. Cryst. 37 (2004) 678-678.

20. Kraus W., Nolze G. POWDER CELL - a program for the representation and manipulation of crystal structures and calculation of the resulting X-ray powder patterns. - J. Appl. Cryst. 29 (1996) 301-303.

21. Downs R.T., Hall-Wallace M. The American mineralogist crystal structure database. - Am. Mineral. 88 (2003) 247-250. http://rruff.geo.arizona.edu/AMS/amcsd.php.

22. Villars P., Cenzual K. (Eds.), Pearson's Crystal Data: Crystal Structure Database for Inorganic Compounds, Release 2014/15, ASM Intern., Materials Park, OH, 2014.

23. Roisnel T., Rodriguez-Carvajal J. WinPLOTR: a Windows tool for powder diffraction patterns analysis. - Mater. Sci. Forum 118 (2001) 378-381.

24. Rachek O.P. X-ray diffraction study of amorphous alloys Al-Ni-Ce-Sc with using Ehrenfest's formula. - J. Non-Cryst. Sol. 352 (2006) 3781-3786.

25. Sarsembinov Sh.Sh., Prikhodko O.Yu., Ryaguzov A.P., Maksimiva S.Ya., Ushanov V.Zh. Atomic structure and short- and medium-range order parameters in amorphous chalcogenide films prepared by different methods. - J. Non-Cryst. Sol. 353 (2007) 2057-2061.

26. De Neufville J.P., Moss S.C., Ovshinsky S.R. Photostructural transformations in amorphous $\mathrm{As}_{2} \mathrm{Se}_{3}$ and $\mathrm{As}_{2} \mathrm{~S}_{3}$ films. - J. Non-Cryst. Sol. 13 (1973/1974) 191-223.

27. Moss S.C., Price D.L. Random packing of structural units and the first sharp diffraction peak in glasses. - In: Physics of disordered materials. Eds. Adler D., Fritsche H., Ovshinsky S.R. Plenum Publ Corp. (1985) 77-95.

28. N'Dri K., Houphouet-Boigny D., Jumas J.-C. Study of first sharp diffraction peak in $\mathrm{As}_{2} \mathrm{~S}_{3}$ glasses by X-ray powder diffraction method. - J. Non-Oxide Glass 3(2012) 29-37.

29. Zeidler A., Salmon P.S. Pressure-driven transformation of the ordering in amorphous networkforming materials. - Phys Rev B 93 (2016) 214204-1-214204-5.

30. Salmon P.S. Real space manifestation of the first sharp diffraction peak in the structure factor of liquid and glassy materials. - Proc. R. Soc. London A 445 (1994) 351-365.

31. Bychkov E., Benmore C.J., Price D.L. Compositional changes in the first sharp diffraction peak in binary selenide glasses, Phys Rev B 72 (2005) 172107-1-4.

32. Golovchak R., Lucas P., Oelgoetz J., Kovalskiy A., York-Winegar J., Saiyasombat Ch., Shpotyuk O., Feygenson M., Neuefeind J., Jain H. Medium range order and structural relaxation in As-Se network glasses through FSDP analysis. - Mater. Chem. Phys. 153 (2015) 432-442. 
I. Implications for the first sharp diffraction peak, photodarkening, and Boson peak. - J. Chem. Phys. 147 (2017) 114505-1-114505-20.

34. Bletry J. Sphere and distance models for binary disordered systems, Phil Mag B62 (1990) 469508.

35. Sozin Yu.I. Diffractometry of coordination spheres. - Crystallography Rep. 39 (1994) 6-13.

36. Feng R., Stachurski Z.H., Rodrigues M.D., Kluth P., Araujo L.L., Bulla D., Ridway M.C. - J. Non-Cryst. Sol. 383 (2013) 21-27.

37. Ehrenfest P. On interference phenomena to be expected when Roentgen rays pass through a diatomic gas. - In: KNAW, Proc. 17 (1914-1915) 1184-1190.

38. Gaskell P.H. The structure of simple glasses: Randomness or pattern the debate goes on. - Glass Phys. Chem. 24 (1998) 180-187.

39. Wright A.C. Crystalline-like ordering in melt-quenched network glasses? - .J Non-Cryst. Sol. 401 (2014) 4-26.

40. Shpotyuk O., Kozdras A., Demchenko P., Shpotyuk Ya., Bujňáková Z., Baláž P. Solid-state amorphization of $\mathrm{As}_{45} \mathrm{~S}_{55}$ alloy induced by high-energy mechanical milling. - Thermochim Acta 642 (2016) 59-66.

41. Baláž P., Baláž M., Shpotyuk O., Demchenko P., Vlček M., Shopska M., Briančin J., Bujňáková Z., Shpotyuk Ya., Selepová B., Balážová L. Properties of arsenic sulphide $\left(\beta-\mathrm{As}_{4} \mathrm{~S}_{4}\right)$ modified by mechanical activation. - J Mater Sci 52 (2017) 1747-1758.

42. Popescu M.A. Non-crystalline chalcogenides. - Kluwer Acad. Publ., Dortrecht, 2000.

43. Stergiou A.C., Rentzeperis P.J. The crystal structure of arsenic selenide, $\mathrm{As}_{2} \mathrm{Se}_{3}-$ Zeitsch Krist. 173 (1985) 185-191.

44. Renninger A.L., Averbach B.L. Crystalline structures of $\mathrm{As}_{2} \mathrm{Se}_{3}$ and $\mathrm{As}_{4} \mathrm{Se}_{4}$. - Acta Cryst. B29 (1973) 1583-1589.

45. Golovchak R., Kovalskiy A., Miller A.C., Jain H., Shpotyuk O. Structure of Se-rich As-Se glasses by high-resolution X-ray photoelectron spectroscopy. - Phys. Rev. B 76 (2007) 125208-1125208-7.

46. Yang G., Bureau B., Rouxel T., Gueguen Y., Gulbiten O., Roiland C., Saidnard E., Yarger J.L., Troles J., Sangleboeuf J.-C., Lucas P. Correlation between structure and physical properties of chalcogenide glasses in the $\mathrm{As}_{\mathrm{x}} \mathrm{Se}_{1-\mathrm{x}}$ system. - Phys. Rev. B 82 (2010) 195206-1-195206-8.

47. Deschamps M., Roiland C., Bureau B., Yang G., Le Polles L., Massiot D. ${ }^{77}$ Se solid-state NMR investigations on $\mathrm{As}_{\mathrm{x}} \mathrm{Se}_{1-\mathrm{x}}$ glasses using CPMG acquisition under MAS. - Soli State Nuclear Magnet. Resonance 40 (2011) 72-77. 
48. Yang G., Gulbiten O., Gueguen Y.., Bureau B., Sangleboeuf J.-C., Roiland C., King E.A., Lucas

P. Fragile-strong behavior in the $\mathrm{As}_{\mathrm{x}} \mathrm{Se}_{1-\mathrm{x}}$ glass forming system in relation to structural dimensionality. - Phys. Rev. B 85 (2012) 144107-1-144107-8.

49. Sykina K., Yang G., Roiland C., Le Polles L., Le Fur E., Pickard C.J., Bureau B., Furet E. ${ }^{77}$ Se solid-state $\mathrm{NMR}$ of $\mathrm{As}_{2} \mathrm{Se}_{3}, \mathrm{As}_{4} \mathrm{Se}_{4}$ and $\mathrm{As}_{4} \mathrm{Se}_{3}$ crystals: a combined experimental and computational study. - Phys. Chem. Chem. Phys. 15 (2013) 6284-6292.

50. Golovchak R., Oelgoetz J., Vlchek M., Esposito A., Saiter A., Saiter J.-M., Jain H. Complex structural rearrangements in As-Se glasses. - J. Chem. Phys. 140 (2014) 054505-1-054505-8.

51. Deschamps M., Genevois C., Cui S., Roiland C., Le Polles L., Furet E., Massiot D., Bureau B. Structure of arsenic selenide glasses studied by NMR: selenium chain length distribution and the Flory model. - J. Phys. Chem. C 119 (2015) 11852-11857.

52. Blachnik Von R., Hoppe A., Wickel U. Die Systeme Arsen-Schwefel und Arsen-Selen und die thermodynamischen Daten ihrer Verbindungen. - Z. Anorg. Allg. Chem. 463 (1980) 78-90.

53. Myers M.B., Felty E.J. Structural characterizations of vitreous inorganic polymers by thermal studies. - Mater. Res. Bull. 2 (1967) 535-546.

54. Shpotyuk O., Hyla M., Boyko V. Compositionally-dependent structural variations in glassy chalcogenides: The case of binary As-Se system. - Comput. Mater. Sci. 110 (2015) 144-151.

55. Leadbetter A.J., Apling A.J. Diffraction studies of glass structure. - J. Non-Cryst. Sol. 15 (1974) 250-268.

56. Wright A.C., Sinclar R.N., Leadbetter A.J. Effect of preparation method on the structure of amorphous solids in the system AS-S. - J. -on-Cryst. Sol. 71 (1985) 295-302.

57. Renninger A.L., Averbach B.L. Atomic radial distribution functions of As-Se glasses. - Phys. Rev. B8 (1973) 1507-1514.

58. Poltavtsev Yu.G. Structure of semiconductors in non-crystalline states. - Usp. Fiz. Nauk. 120 (1976) 581-612.

59. Tanaka K. High-pressure structural changes in chalcogenide glasses. - Solid State Commun. 58 (1986) 469-471.

60. Tanaka K. Pressure dependence of the first sharp diffraction peak in chalcogenide and oxide glasses. - Phil. Mag. Lett. 57 (1988) 183-187.

61. Cherin P., Unger P. The crystal structure of trigonal selenium. - Inorg. Chem. 6 (1967) 15891591.

62. Price D.L., Moss S.C., Reijers R., Saboungi M.-L., Susman S. Intermediate-range order in glasses and liquids. - J. Phys.: Condens. Matter 1 (1989) 1005-1008.

63. Popescu M. Medium range order in chalcogenide glasses. - In: Phys. Appl. Non-Cryst. Semicond. in Optoelectronics. Eds. Andriesh A. and Bertolotti M. Kluwer Acad Publ. DordrechtBoston-London, 1997, 215-232 
64. Mazets T.F., Smorgonskaya E.A., Tikhomirov V.K. Stable anisotropy in glassy $\mathrm{As}_{2} \mathrm{~S}_{3}: \mathrm{In}$. - J. Non-Cryst. Sol. 164-166 (1993) 1215-1218.

65. Vateva E., Savova E. New medium-range order features in Ge-Sb-S glasses. - J. Non-Cryst. Sol. 192-193 (1995) 145-148.

66. Baidakova M.V., Faleev N.N., Mazets T.F., Smorgonskaya E.A. Nano-scale medium-range order in semiconducting glassy chalcogenides. - J. Non-Cryst. Sol. 192-193 (1995) 149-152.

67. Arsova D., Vateva E., Skordeva E., Petkov V. New features of the medium range order in $\mathrm{Ge}_{\mathrm{x}} \mathrm{As}_{40-\mathrm{x}} \mathrm{Se}_{60}$ glasses. - Solid State Commun. 98 (1996) 595-598.

68. Tanaka Ke. Photoexpansion in $\mathrm{As}_{2} \mathrm{~S}_{3}$ glass. - Phys. Rev. B 57 (1998) 5163-5167.

69. Shpotyuk O., Kozyukhin S., Shpotyuk Ya., Demchenko P., Mitsa V., Veres M. Coordination disordering in near-stoichiometric arsenic sulfide glass. - J. Non-Cryst. Sol. 402 (2014) 236-243.

70. Shpotyuk O., Calvez L., Petracovschi E., Klym H., Ingram A., Demchenko P. Thermallyinduced crystallization behaviour of $80 \mathrm{GeSe}_{2}-20 \mathrm{Ga}_{2} \mathrm{Se}_{3}$ glass as probed by combined X-ray diffraction and PAL spectroscopy. - J. Alloys Compounds 582 (2014) 323-327.

71. Klym H., Ingram A., Shpotyuk O., Calvez L., Petracovschi E., Kulyk B., Serkiz R., Szatanik R. "Cold" crystallization in nanostructurized $80 \mathrm{GeSe}_{2}-20 \mathrm{Ga}_{2} \mathrm{Se}_{3}$ glass. - Nanoscale Res. Lett. 10 (2015) 49-1-49-8.

72. Shpotyuk Ya., Ingram A., Shpotyuk O., Dziedzic A., Boussard-Pledel C., Bureau B. Freevolume nanostructurization in Ga-modified $\mathrm{As}_{2} \mathrm{Se}_{3}$ glass. - Nanoscale Res. Lett. 11 (2016) 20-1-7. 73. Shpotyuk O., Kozdras A., Demchenko P., Shpotyuk Ya., Bujňáková Z., Baláž P. Solid-state amorphization of $\mathrm{As}_{45} \mathrm{~S}_{55}$ alloy induced by high-energy mechanical milling. - Thermochimica Acta 642 (2016) 59-66. 
Fig. 1. Typical particle size distribution in $\mathrm{g}-\mathrm{As}_{40} \mathrm{Se}_{60}$ affected to high-energy MM.

Fig. 2. Experimental (red points) and calculated (black solid line) XRPD profiles for unmilled g$\mathrm{As}_{5} \mathrm{Se}_{95}$ (a), g-As ${ }_{10} \mathrm{Se}_{90}$ (b), g-As ${ }_{20} \mathrm{Se}_{80}$ (c), g-As ${ }_{29} \mathrm{Se}_{71}$ (d), g-As ${ }_{35} \mathrm{Se}_{65}$ (e), g-As $\mathrm{As}_{40} \mathrm{Se}_{60}$ (f) showing the FSDP around $12-25^{\circ} 2 \Theta$ (the difference curve is given at the bottom by blue solid line).

Fig. 3. Experimental (red points) and calculated (black solid line) XRPD profiles for milled g$\mathrm{As}_{5} \mathrm{Se}_{95}$ (a), g-As ${ }_{10} \mathrm{Se}_{90}$ (b), g-As ${ }_{20} \mathrm{Se}_{80}$ (c), g-As ${ }_{29} \mathrm{Se}_{71}$ (d), g-As ${ }_{35} \mathrm{Se}_{65}$ (e), g- $\mathrm{As}_{40} \mathrm{Se}_{60}$ (f) showing the FSDP around $12-25^{\circ} 2 \Theta$ (the difference is given at the bottom by blue solid line).

Fig. 4. Low- $Q$ part of the reduced X-ray structure factor $F(Q)=Q(S(Q)-1)$ for milled $\mathrm{g}-\mathrm{As}_{\mathrm{x}} \mathrm{Se}_{100 \text {-x }}$ (the $\mathrm{x}$ parameter is denoted under the curve, which is offset along the $y$ axis, for clarity). Slight shoulder at $Q \cong \sim 1.5 \AA^{-1}$ producing the FSDP asymmetry is visible (see text for more details).

Fig. 5. Experimental XRPD profiles for unmilled (green curve) and milled (black curve) g-As $\mathrm{As}_{40} \mathrm{Se}_{60}$ as compared with theoretical Bragg diffraction reflexes for monoclinic $\mathrm{As}_{2} \mathrm{Se}_{3}$ (red lines) and trigonal Se (blue lines). The most intense diffraction lines of these crystals are denoted, slight shoulder around $\sim 20-22^{\circ} 2 \theta$ is detectable in both $\mathrm{g}-\mathrm{As}_{40} \mathrm{Se}_{60}$ samples (see text for more details). 


\begin{tabular}{|l|l|l|l|l|}
\hline $\boldsymbol{x}$ & $\boldsymbol{T}_{\boldsymbol{a}}$ & $\boldsymbol{\rho}, \mathbf{g} \cdot \mathbf{c m}^{-3}$ & $\boldsymbol{T}_{\boldsymbol{g}},{ }^{\boldsymbol{o}} \boldsymbol{C}$ & $\boldsymbol{l}_{\boldsymbol{s}}, \mathbf{A}$ \\
\hline 5 & $50{ }^{\circ} \mathrm{C}$ & 4.314 & 65 & 3.87 \\
\hline 10 & $60{ }^{\circ} \mathrm{C}$ & 4.348 & 81 & 3.86 \\
\hline 15 & $65{ }^{\circ} \mathrm{C}$ & 4.385 & 92 & 3.84 \\
\hline 17.5 & $70{ }^{\circ} \mathrm{C}$ & 4.402 & 96 & 3.83 \\
\hline 20 & $75^{\circ} \mathrm{C}$ & 4.420 & 102 & 3.83 \\
\hline 22.5 & $80{ }^{\circ} \mathrm{C}$ & 4.444 & 106 & 3.82 \\
\hline 25 & $90{ }^{\circ} \mathrm{C}$ & 4.468 & 112 & 3.81 \\
\hline 29 & $115^{\circ} \mathrm{C}$ & 4.503 & 124 & 3.80 \\
\hline 33 & $130{ }^{\circ} \mathrm{C}$ & 4.546 & 138 & 3.78 \\
\hline 35 & $140{ }^{\circ} \mathrm{C}$ & 4.567 & 148 & 3.78 \\
\hline 37 & $150{ }^{\circ} \mathrm{C}$ & 4.593 & 163 & 3.77 \\
\hline 40 & $160{ }^{\circ} \mathrm{C}$ & 4.605 & 184 & 3.77 \\
\hline
\end{tabular}


Table 2. The FSDP parameterization in unmilled and milled $\mathrm{g}-\mathrm{As}_{\mathrm{x}} \mathrm{Se}_{100-\mathrm{x}}$.

\begin{tabular}{|c|c|c|c|c|c|c|c|c|c|c|}
\hline \multirow{2}{*}{$\begin{array}{l}\text { Sample } x \text {, } \\
\text { state }\end{array}$} & $2 \theta$ & $F W H M$ & $Q$ & $\Delta Q$ & $R$ & $\Delta R / R_{0}$ & $L$ & $\Delta L / L_{0}$ & $d_{s}$ & $\Delta d_{s} / d_{s} 0$ \\
\hline & ${ }^{\circ} 2 \theta$ & ${ }^{o} 2 \theta$ & $\AA^{-1}$ & $\AA^{-1}$ & $\AA$ & $\%$ & $\AA$ & $\%$ & $\AA$ & $\%$ \\
\hline $\mathrm{x}=40$; unmilled & $18.014(13)$ & $6.02(4)$ & 1.28 & 0.43 & 4.92 & - & 14.67 & - & 6.05 & - \\
\hline $\mathrm{x}=40 ;$ milled & $17.862(11)$ & $6.02(3)$ & 1.27 & 0.43 & 4.96 & +0.8 & 14.65 & -0.1 & 6.10 & 0.8 \\
\hline $\mathrm{x}=37 ;$ unmilled & $18.251(13)$ & $6.20(4)$ & 1.29 & 0.44 & 4.86 & - & 14.26 & - & 5.97 & - \\
\hline $\mathrm{x}=37 ;$ milled & $18.133(13)$ & $6.33(4)$ & 1.28 & 0.45 & 4.89 & +0.6 & 13.96 & -2.1 & 6.01 & 0.7 \\
\hline $\mathrm{x}=35 ;$ unmilled & $18.355(13)$ & $6.44(4)$ & 1.30 & 0.46 & 4.83 & - & 13.72 & - & 5.94 & - \\
\hline $\mathrm{x}=35 ;$ milled & $18.231(14)$ & $6.60(4)$ & 1.29 & 0.47 & 4.86 & +0.6 & 13.38 & -2.5 & 5.98 & 0.7 \\
\hline $\mathrm{x}=33$; unmilled & $18.423(14)$ & $6.25(4)$ & 1.31 & 0.44 & 4.81 & - & 14.14 & - & 5.92 & - \\
\hline $\mathrm{x}=33 ;$ milled & $18.369(15)$ & $6.70(5)$ & 1.30 & 0.48 & 4.83 & +0.4 & 13.18 & -6.8 & 5.94 & 0.3 \\
\hline $\mathrm{x}=29$; unmilled & $18.697(14)$ & $6.47(4)$ & 1.32 & 0.46 & 4.74 & - & 13.65 & - & 5.83 & - \\
\hline $\mathrm{x}=29 ;$ milled & $18.633(14)$ & $6.81(4)$ & 1.32 & 0.48 & 4.76 & +0.4 & 12.97 & -5.0 & 5.85 & 0.3 \\
\hline$x=25$; unmilled & $18.977(17)$ & $6.43(5)$ & 1.34 & 0.46 & 4.67 & - & 13.73 & - & 5.75 & - \\
\hline $\mathrm{x}=25 ;$ milled & $18.917(16)$ & $6.84(4)$ & 1.34 & 0.49 & 4.69 & +0.4 & 12.91 & -6.0 & 5.77 & 0.3 \\
\hline$x=22.5$; unmilled & $19.110(22)$ & $6.64(6)$ & 1.35 & 0.47 & 4.64 & - & 13.30 & - & 5.71 & - \\
\hline$x=22.5 ;$ milled & $19.092(19)$ & $7.04(5)$ & 1.35 & 0.50 & 4.64 & 0 & 12.54 & -5.7 & 5.71 & 0 \\
\hline$x=20$; unmilled & $19.323(21)$ & $6.80(9)$ & 1.37 & 0.48 & 4.59 & - & 13.00 & - & 5.65 & - \\
\hline$x=20 ;$ milled & $19.236(27)$ & $6.58(7)$ & 1.36 & 0.47 & 4.61 & +0.4 & 13.43 & +3.3 & 5.67 & 0.4 \\
\hline$x=17.5$; unmilled & $19.490(21)$ & $6.31(5)$ & 1.38 & 0.45 & 4.55 & - & 13.99 & - & 5.60 & - \\
\hline$x=17.5$; milled & $19.532(22)$ & $6.26(5)$ & 1.38 & 0.45 & 4.54 & -0.2 & 14.10 & +0.8 & 5.58 & -0.4 \\
\hline $\mathrm{x}=15$; unmilled & $19.621(22)$ & $6.03(5)$ & 1.39 & 0.43 & 4.52 & - & 14.64 & - & 5.56 & - \\
\hline $\mathrm{x}=15 ;$ milled & $19.597(24)$ & $6.00(5)$ & 1.39 & 0.43 & 4.53 & +0.2 & 14.71 & +0.5 & 5.57 & 0.2 \\
\hline $\mathrm{x}=10$; unmilled & $19.962(28)$ & $5.54(6)$ & 1.41 & 0.39 & 4.44 & - & 15.94 & - & 5.46 & - \\
\hline $\mathrm{x}=10 ;$ milled & $19.901(26)$ & $5.58(6)$ & 1.41 & 0.40 & 4.46 & +0.5 & 15.83 & -0.7 & 5.48 & 0.4 \\
\hline $\mathrm{x}=5$; unmilled & $20.011(5)$ & $4.51(13)$ & 1.42 & 0.32 & 4.43 & - & 19.56 & - & 5.45 & - \\
\hline $\mathrm{x}=5$; milled & $20.101(27)$ & $4.57(8)$ & 1.42 & 0.33 & 4.41 & -0.5 & 19.31 & -1.3 & 5.42 & -0.5 \\
\hline
\end{tabular}




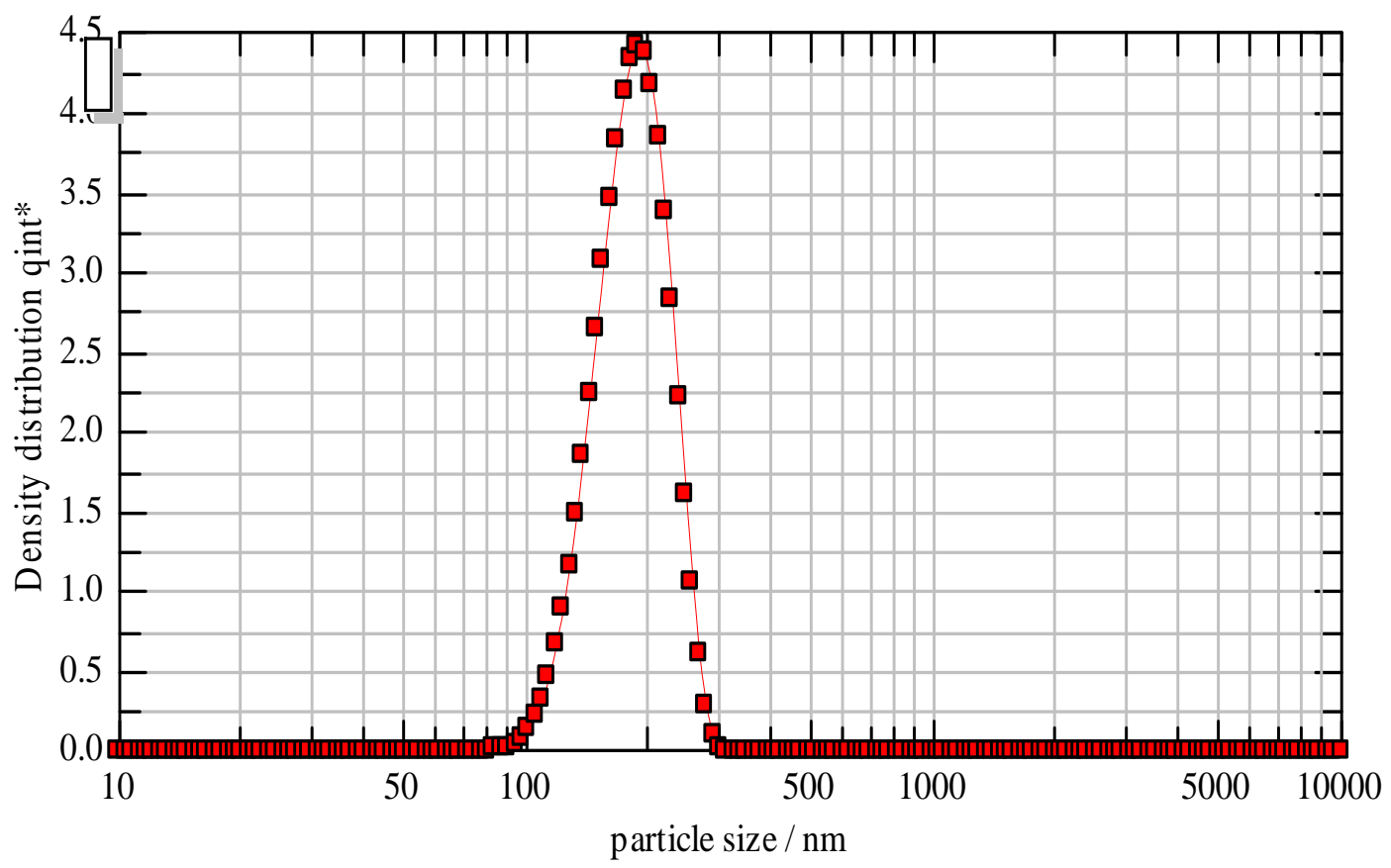

Fig. 1. Typical particle size distribution in $\mathrm{g}-\mathrm{As}_{40} \mathrm{Se}_{60}$ affected to high-energy MM. 


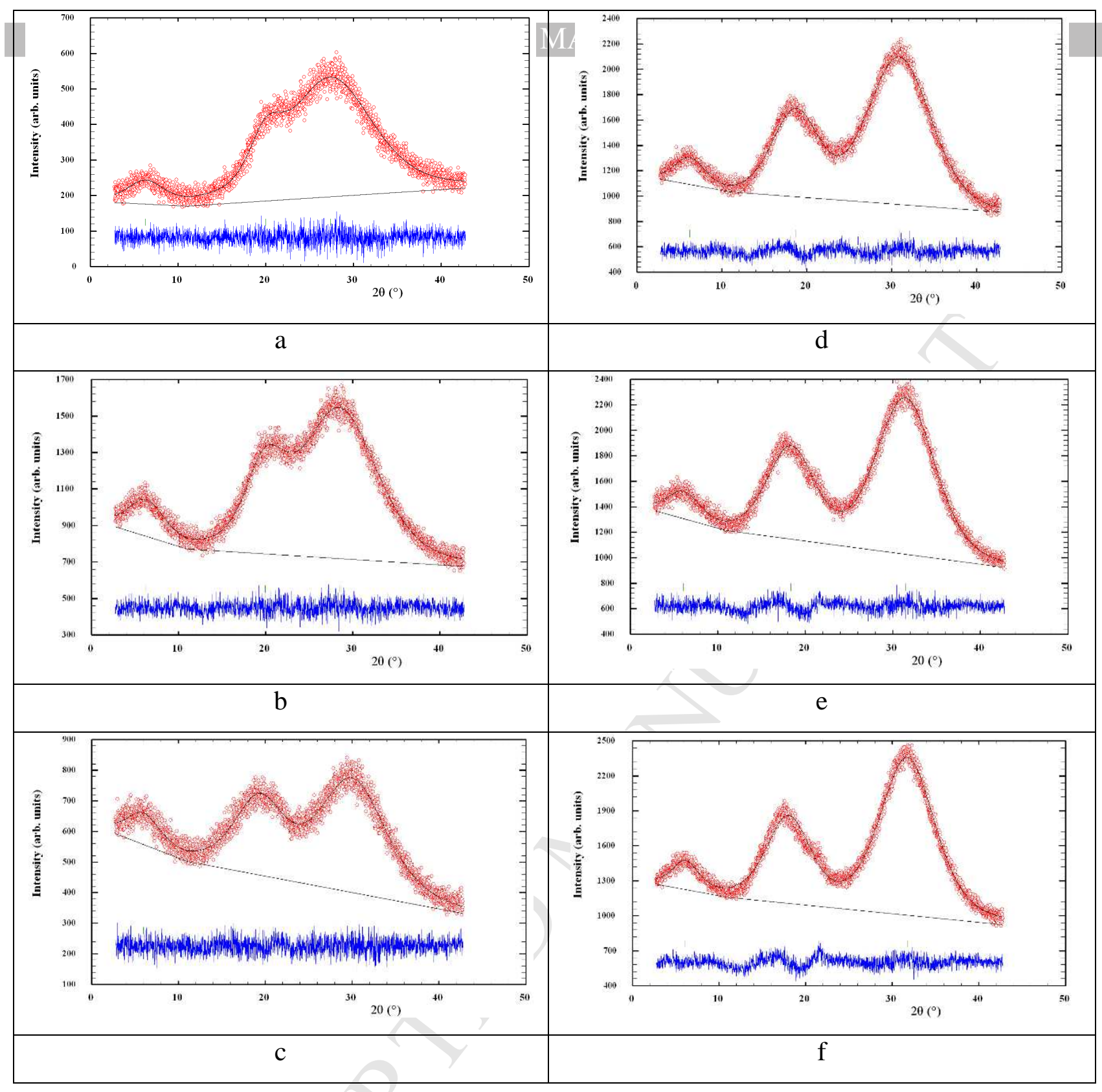

Fig. 2. Experimental (red points) and calculated (black solid line) XRPD profiles for unmilled g$\mathrm{As}_{5} \mathrm{Se}_{95}$ (a), g-As ${ }_{10} \mathrm{Se}_{90}$ (b), g-As ${ }_{20} \mathrm{Se}_{80}$ (c), g-As ${ }_{29} \mathrm{Se}_{71}$ (d), g-As ${ }_{35} \mathrm{Se}_{65}$ (e), g-As ${ }_{40} \mathrm{Se}_{60}$ (f) showing the FSDP around $12-25^{\circ} 2 \Theta$ (the difference curve is given at the bottom by blue solid line). 


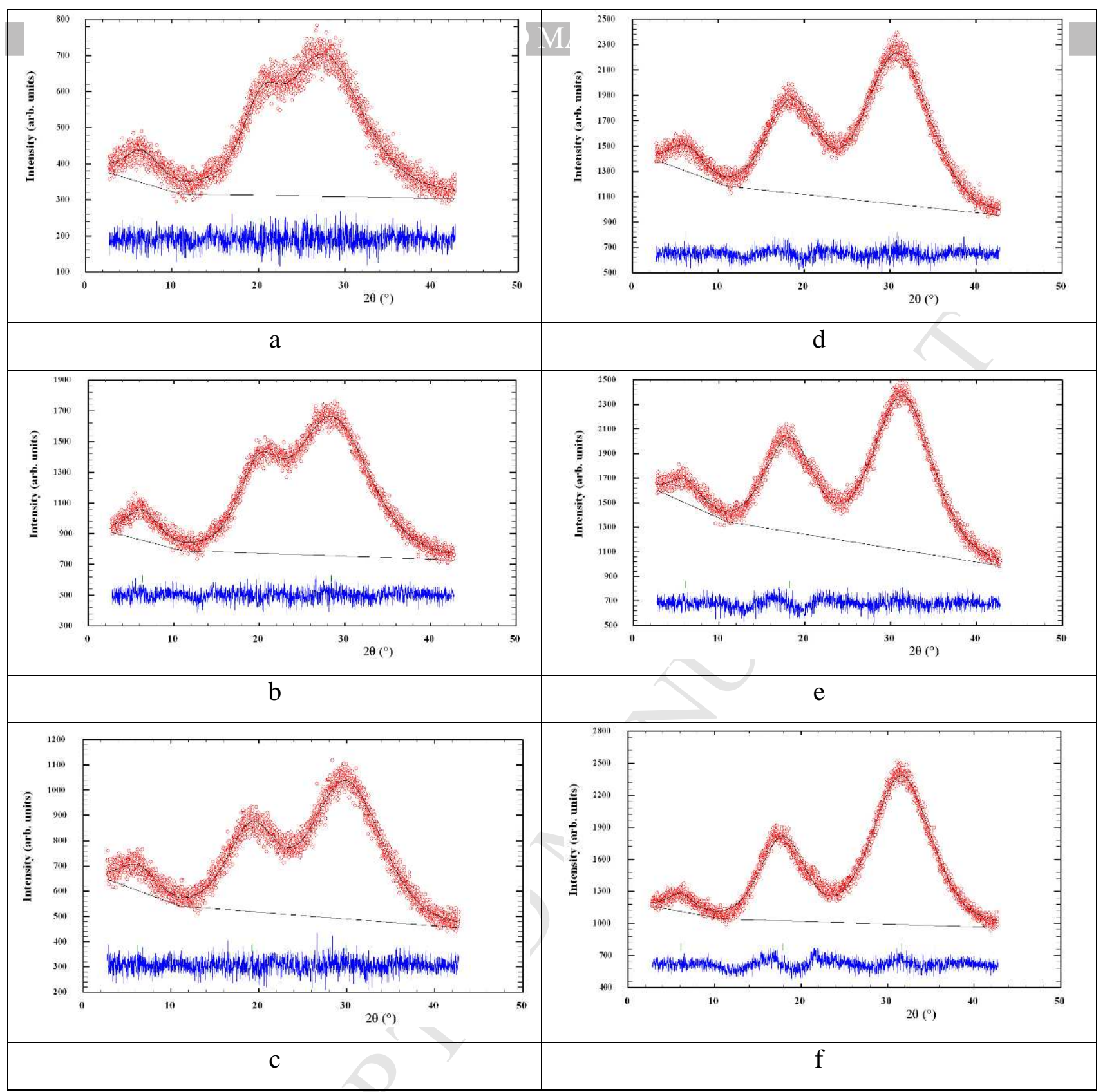

Fig. 3. Experimental (red points) and calculated (black solid line) XRPD profiles for milled g$\mathrm{As}_{5} \mathrm{Se}_{95}$ (a), g-As ${ }_{10} \mathrm{Se}_{90}$ (b), g-As ${ }_{20} \mathrm{Se}_{80}$ (c), g-As $\mathrm{As}_{29} \mathrm{Se}_{71}$ (d), g-As ${ }_{35} \mathrm{Se}_{65}$ (e), g-As $\mathrm{As}_{40} \mathrm{Se}_{60}$ (f) showing the FSDP around $12-25^{\circ} 2 \Theta$ (the difference is given at the bottom by blue solid line). 


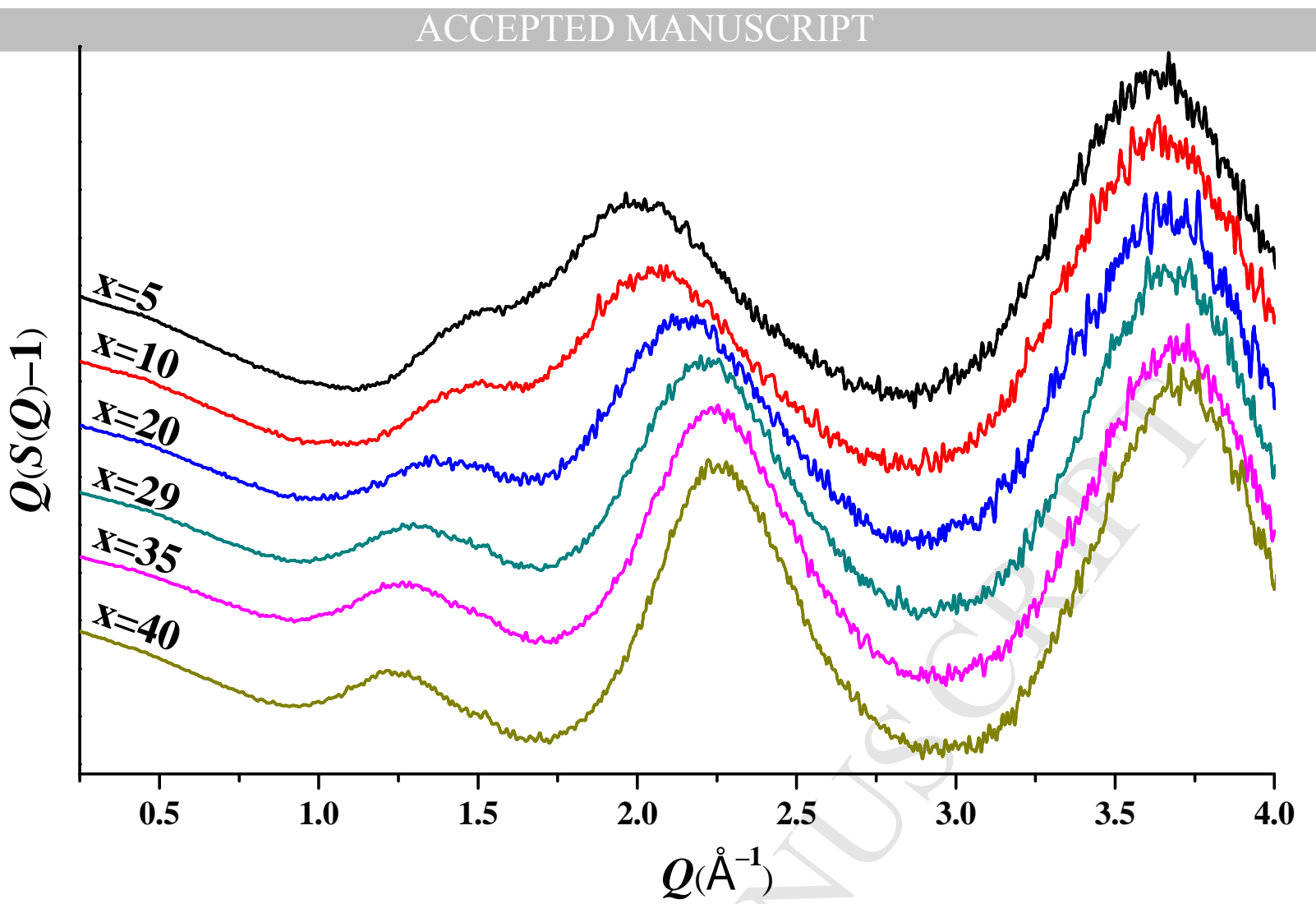

Fig. 4. Low- $Q$ part of the reduced X-ray structure factor $F(Q)=Q(S(Q)-1)$ for milled $\mathrm{g}-\mathrm{As}_{\mathrm{x}} \mathrm{Se}_{100-\mathrm{x}}$ (the $\mathrm{x}$ parameter is denoted under the curve, which is offset along the $y$ axis, for clarity).

Slight shoulder at $Q \cong \sim 1.5 \AA^{-1}$ producing the FSDP asymmetry is visible (see text for more details). 


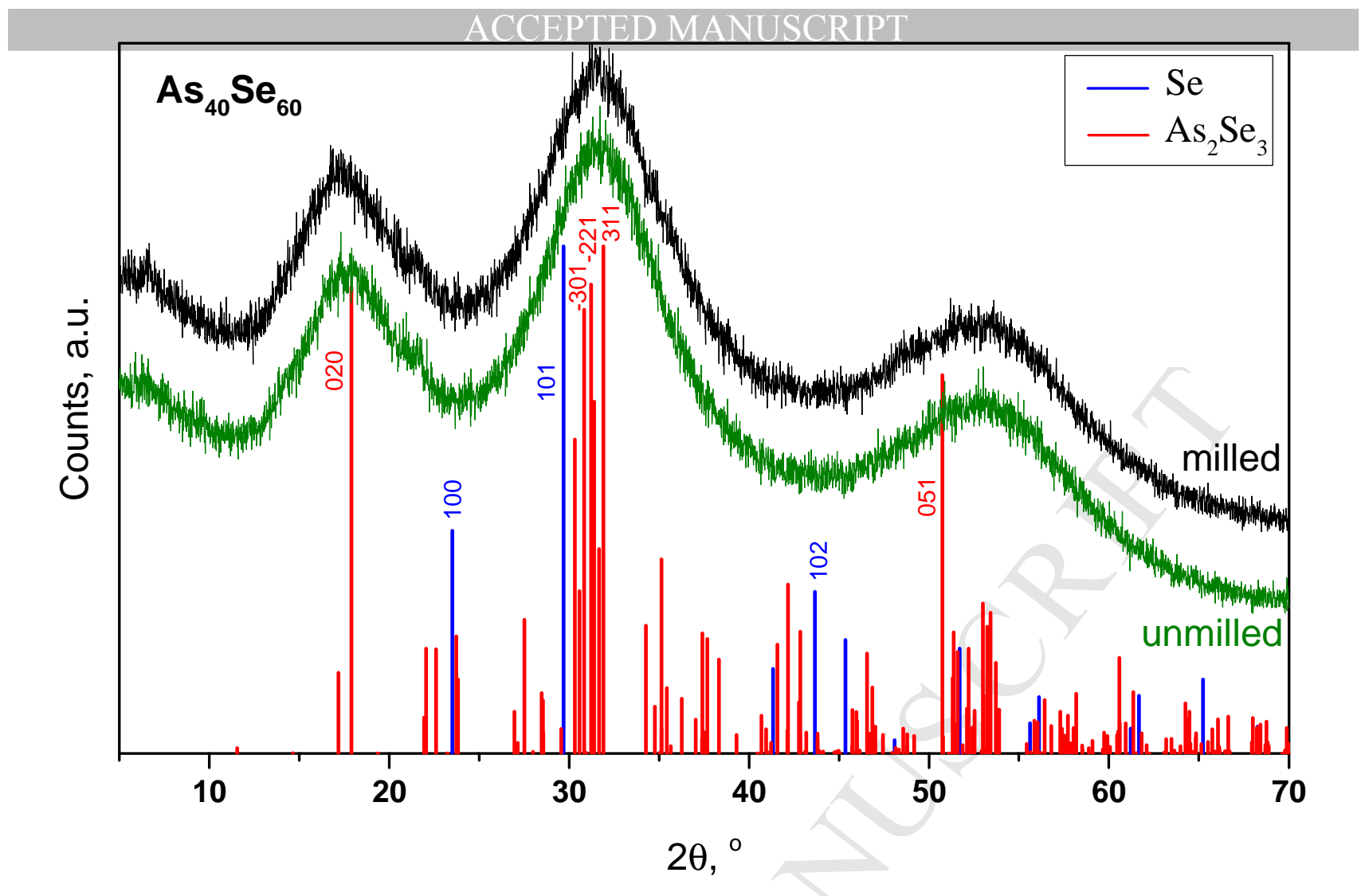

Fig. 5. Experimental XRPD profiles for unmilled (green curve) and milled (black curve) g-As $\mathrm{As}_{40} \mathrm{Se}_{60}$ as compared with theoretical Bragg diffraction reflexes for monoclinic $\mathrm{As}_{2} \mathrm{Se}_{3}$ (red lines) and trigonal Se (blue lines). The most intense diffraction lines of these crystals are denoted, slight shoulder around $\sim 20-21^{\circ} 2 \theta$ is detectable in both $\mathrm{g}-\mathrm{As}_{40} \mathrm{Se}_{60}$ samples (see text for more details). 


\section{Highlights}

Intermediate-range ordering in $\mathrm{As}_{\mathrm{x}} \mathrm{Se}_{100-\mathrm{x}}$ glasses modified under high-energy milling FSDP position and width are milling-invariant in Se-rich $\mathrm{As}_{\mathrm{x}} \mathrm{Se}_{100-\mathrm{x}}$ glasses $(\mathrm{x}<20)$ Increased FSDP width in glassy $\mathrm{As}_{\mathrm{x}} \mathrm{Se}_{100-\mathrm{x}}(20 \leq \mathrm{x} \leq 40)$ due to milling-derived defects 